\title{
Effect of disorder on the thermal transport and elastic properties in thermoelectric $\mathbf{Z n}_{4} \mathbf{S b}_{3}$
}

\author{
S. Bhattacharya,* R. P. Hermann, ${ }^{\dagger}$ and V. Keppens \\ Materials Science \& Engineering, The University of Tennessee, Knoxville, Tennessee 37996, USA \\ T. M. Tritt \\ Department of Physics, Clemson University, Clemson, South Carolina 29634, USA \\ G. J. Snyder \\ California Institute of Technology, Pasadena, California 91125, USA \\ (Received 7 June 2006; revised manuscript received 1 August 2006; published 17 October 2006)
}

\begin{abstract}
$\mathrm{Zn}_{4} \mathrm{Sb}_{3}$ undergoes a phase transition from $\alpha$ to $\beta$ phase at $T_{1} \approx 250 \mathrm{~K}$. The high temperature $\beta$ - $\mathrm{Zn}_{4} \mathrm{Sb}_{3}$ phase has been widely investigated as a potential state-of-the-art thermoelectric (TE) material, due to its remarkably low thermal conductivity. We have performed electronic and thermal transport measurements exploring the structural phase transition at $250 \mathrm{~K}$. The $\alpha$ to $\beta$ phase transition manifests itself by anomalies in the resistivity, thermopower, and specific heat at $250 \mathrm{~K}$ as well as by a reduction in the thermal conductivity as $\mathrm{Zn}_{4} \mathrm{Sb}_{3}$ changes phase from the ordered $\alpha$ to the disordered $\beta$-phase. Moreover, measurements of the elastic constants using resonant ultrasound spectroscopy (RUS) reveal a dramatic softening at the order-disorder transition upon warming. These measurements provide further evidence that the remarkable thermoelectric properties of $\beta-\mathrm{Zn}_{4} \mathrm{Sb}_{3}$ are tied to the disorder in the crystal structure.
\end{abstract}

DOI: 10.1103/PhysRevB.74.134108

\section{INTRODUCTION}

$\mathrm{Zn}_{4} \mathrm{Sb}_{3}$ has attracted much attention in the recent years, not only for its promising thermoelectric properties, ${ }^{1}$ but also for its spectacular thermodynamic properties. The complex $\mathrm{Zn}_{4} \mathrm{Sb}_{3}$ structure is known to undergo two distinct phase transitions upon warming, first from the $\alpha$ to $\beta$ phase at $T_{1} \approx 250 \mathrm{~K}$ and second from the $\beta$ to $\gamma$ phase at $T_{2} \approx 765 \mathrm{~K}^{2}{ }^{2}$ Explored in the early 1900 's, ${ }^{3,4}$ the $\mathrm{Zn}_{4} \mathrm{Sb}_{3}$ phase diagram ${ }^{5,6}$ was reinspected by Mayer et al. $^{7}$ in 1978. $\mathrm{X}$-ray diffraction studies show an $R$-centered hexagonal unit cell for $\beta-\mathrm{Zn}_{4} \mathrm{Sb}_{3}$ above $250 \mathrm{~K}$, which changes into a $C$-centered monoclinic unit cell for $\alpha-\mathrm{Zn}_{4} \mathrm{Sb}_{3}$ below the transition temperature $T_{1}{ }^{8}$ This research focuses on the elastic and thermal transport properties related to the lower temperature $\alpha$ to $\beta$ phase transition at $T_{1} \approx 250 \mathrm{~K}$.

The low-temperature $C$-centered monoclinic unit cell of $\alpha-\mathrm{Zn}_{4} \mathrm{Sb}_{3}$ contains $26 \mathrm{Zn}$ and $20 \mathrm{Sb}$ atoms leading to $\mathrm{Zn}_{3.9} \mathrm{Sb}_{3}$ (or $\mathrm{Zn}_{13} \mathrm{Sb}_{10}$ ) stoichiometry. However, above $250 \mathrm{~K}, \beta-\mathrm{Zn}_{4} \mathrm{Sb}_{3}$ exhibits a rhombohedral (or $R$-centered hexagonal) unit cell enclosing $36 \mathrm{Zn}^{2+}, 18 \mathrm{Sb}^{3-}$, and $12 \mathrm{Sb}^{2-}$ ions resulting in $\mathrm{Zn}_{3.6} \mathrm{Sb}_{3}$ (or $\mathrm{Zn}_{36} \mathrm{Sb}_{30}$ ) stoichiometry. This anomaly in stoichiometry between $\alpha\left(\mathrm{Zn}_{3.9} \mathrm{Sb}_{3}\right)$ and $\beta\left(\mathrm{Zn}_{3.6} \mathrm{Sb}_{3}\right)$ has recently been solved by Snyder et al. ${ }^{9}$ and Cargnoni et al. ${ }^{10}$ with the discovery of interstitial $\mathrm{Zn}$ atoms in the $\beta$ phase resulting in a total of $39 \mathrm{Zn}^{2+}$ ions (per rhombohedral cell) required for charge compensation. Snyder et $a l .{ }^{9}$ have also pointed out that the sample density and chemical composition are in agreement with the experimental values after the inclusion of the three interstitial $\mathrm{Zn}$ atoms. The unusually low thermal conductivity in the $\beta-\mathrm{Zn}_{4} \mathrm{Sb}_{3}$ has been associated with the disorder in the structure of $\beta-\mathrm{Zn}_{4} \mathrm{Sb}_{3}$, due to the occurrence of these interstitial $\mathrm{Zn}$ atoms. Maximum entropy method (MEM) electron density analysis by Cargnoni et al. ${ }^{10}$ points to a possible $\mathrm{Sb}$ disorder along the $c$ axis.
PACS number(s): 73.50.Lw, 72.15.Jf, 72.15.Eb, 72.80.Ga

$\beta-\mathrm{Zn}_{4} \mathrm{Sb}_{3}$ is a prospective $p$-type thermoelectric material that may provide an improvement in efficiency compared to the state-of-the-art thermoelectric material TAGS $(\mathrm{AgSbTe})_{0.15}(\mathrm{GeTe})_{0.85} \cdot \quad \beta-\mathrm{Zn}_{4} \mathrm{Sb}_{3}$ exhibits the "phononglass electron-crystal" properties of an ideal thermoelectric material, with an unusually low thermal conductivity. This leads to a promising thermoelectric figure-of-merit, $Z T \approx 1.3$ at $673 \mathrm{~K}$ in $\beta-\mathrm{Zn}_{4} \mathrm{Sb}_{3} \cdot{ }^{1} \mathrm{Zn}_{4} \mathrm{Sb}_{3}$ also exhibits a comparatively high thermopower, typical of semiconductors, combined with a metallic-like resistivity increasing linearly from room temperature to about $600 \mathrm{~K}$. Kim and Singh ${ }^{11}$ have investigated this unconventional behavior of the electronic transport in $\mathrm{Zn}_{4} \mathrm{Sb}_{3}$ using first-principles calculation of the band structure. They have attributed the high thermopower to the "complex" and "energy-dependent" Fermi surface. ${ }^{11}$ $\beta-\mathrm{Zn}_{4} \mathrm{Sb}_{3}$ is thus a "low carrier density" material with a relatively high thermopower and a good carrier mobility.

\section{EXPERIMENTAL PROCEDURE}

$\mathrm{Zn}_{3.97} \mathrm{Sb}_{3}$ was prepared by direct reaction of the elements. The $\mathrm{Zn}_{3.97} \mathrm{Sb}_{3}$ stoichiometry is in the middle of the experimental stability range for $\mathrm{Zn}_{3.9} \mathrm{Sb}_{3}$ and was chosen to ensure that the material is single phased. The stoichiometric mixture was enclosed in a fused silica ampoule that was flame sealed under dynamic vacuum ( $10^{-6}$ Torr). The sample was briefly melted above $923 \mathrm{~K}$ and lightly shaken to ensure homogeneity of the liquid, and then water quenched. Finally, the sample was annealed at $573 \mathrm{~K}$ for two days before opening the ampoule. The phase purity of the sample was checked by powder x-ray diffraction and scanning electron microscopy with energy dispersive spectroscopy. No traces of $\mathrm{ZnSb}$ or $\mathrm{Zn}$ were found by either method. The sample was then ball milled, hot pressed at $623 \mathrm{~K}$ and sliced with a diamond saw.

Electrical resistivity and thermopower are measured simultaneously in a closed cycle refrigerator from 10 to $300 \mathrm{~K}$ 
using a custom designed sample mount. ${ }^{12}$ The thermoelectric voltages and the temperature gradient are determined by soldering the sample between two copper blocks with a differential thermocouple and a heater and by measuring the appropriate voltages. Resistivity is measured using the standard four-probe technique and the direction of current is reversed to subtract any thermal voltages. The thermal conductivity is measured using a steady state technique from 10 to $300 \mathrm{~K}$ using a custom designed system. ${ }^{13}$ The sample is soldered to a stable temperature copper base with a differential thermocouple attached on two no. 38 copper wires attached to the sample. The thermal conductance is measured by power vs $\Delta T$ sweeps performed at each temperature. The power is provided by a strain gauge (100 ohm heater) attached to the top of the sample while the $\Delta T$ is measured by a calibrated differential thermocouple. Precise measurements of the dimensions of the sample and proper precautions to avoid heat losses through conduction, convection, and radiation lead to typical accuracies in the thermal conductivity of about 5-7\%. Specific heat and Hall effect measurements are performed using the commercial Quantum Design Physical Properties Measurement System (PPMS). The specific heat is measured between 2 and $300 \mathrm{~K}$ by the relaxation technique. We have performed a 5-wire Hall measurement using the PPMS, where 3 voltage leads are balanced to nullify any offset due to the sample resistance in the absence of a magnetic field. The elastic moduli are measured using resonant ultrasound spectroscopy (RUS). ${ }^{14-16}$ In the RUS experiment, the mechanical resonances of a freely vibrating solid of known shape are measured, and an iteration procedure is used to "match" the measured lines with the calculated spectrum. This allows determination of all elastic constants of the solid from a single frequency scan, which clearly indicates a main advantage of RUS: there is no need for separate measurements to probe different moduli, and multiple sample remounts and temperature sweeps are avoided. Another advantage lies in the ability of RUS to work with small samples: whereas conventional techniques can demand a sample size up to a centimeter, RUS measurements can be made on $\mathrm{mm}$-sized samples. Measurements as a function of temperature were performed using a home-build probe that fits in the PPMS.

\section{RESULTS AND DISCUSSION}

The resistivity in $\mathrm{Zn}_{4} \mathrm{Sb}_{3}$, shown in Fig. 1(a), exhibits a unique temperature dependence. As the sample is cooled down from room temperature, an anomalous peak is observed at about $250 \mathrm{~K}$, indicating the $\alpha$ - $\beta$ phase transition in $\mathrm{Zn}_{4} \mathrm{Sb}_{3}{ }^{17,18}$ The plateau in resistivity, observed between $230 \mathrm{~K}$ and $140 \mathrm{~K}$, may be due to the effect of an impurity scattering process, arising from a phase impurity caused by a "premature effect" of the order-disorder phase transition at $250 \mathrm{~K}$. A second phase transition at $T \approx 234 \mathrm{~K}$ has been reported by another group. ${ }^{19}$ The slope in resistivity changes below $140 \mathrm{~K}$, decreasing with decrease in temperature in a semimetallic manner. The room temperature resistivity is somewhat higher than that reported in Ref. 1.

The thermopower in $\mathrm{Zn}_{4} \mathrm{Sb}_{3}$, shown in Fig. 1(b), is positive, with a magnitude of $160 \mu \mathrm{V} / \mathrm{K}$ at room temperature,
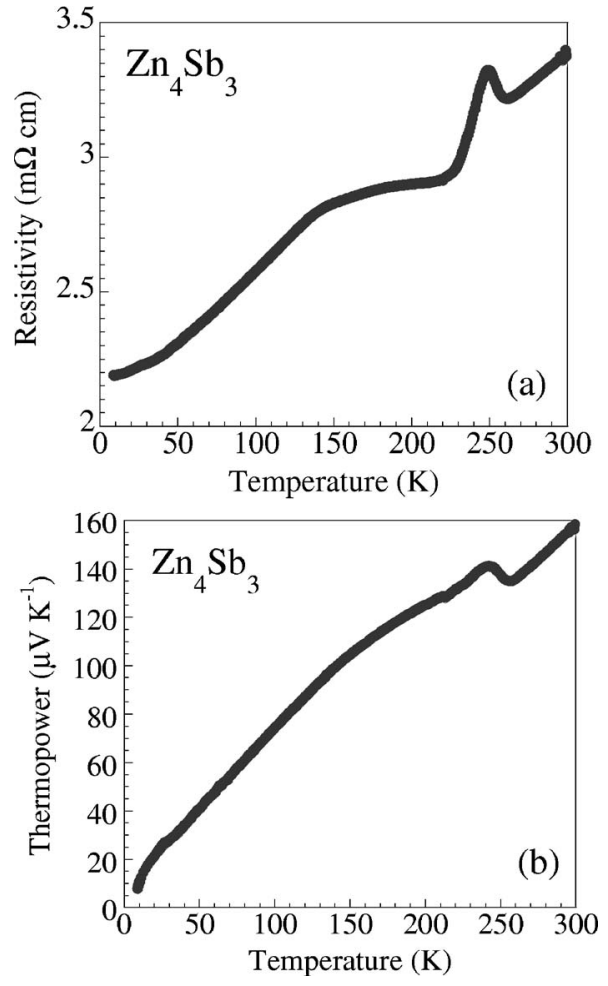

FIG. 1. (a) Resistivity and (b) Thermopower of $\mathrm{Zn}_{4} \mathrm{Sb}_{3}$ as a function of temperature from 10 to $300 \mathrm{~K}$. The structural phase transition is indicated by the peak at $T_{1} \approx 250 \mathrm{~K}$.

higher than that reported in Ref. 1. A well-defined peak is observed at $250 \mathrm{~K}$ that reciprocates the peak in resistivity. Thermopower is diffusive in nature, with no significant phonon drag peak, ${ }^{20}$ increasing monotonically with increase in temperature to about $140 \mathrm{~K}$. A slight bend or change in slope is observed in the thermopower at about $140 \mathrm{~K}$, consistent with the plateau observed in the resistivity at that temperature.

The Hall measurements of $\mathrm{Zn}_{4} \mathrm{Sb}_{3}$ indicate a hole concentration, $p \approx 3 \times 10^{19} \mathrm{~cm}^{-3}$, within the uncertainty of measurement with little temperature dependence across the temperature range measured. The material reported in Ref. 1 has a higher carrier concentration (by at least a factor of 3) than the sample used in this study which explains the lower resistivity, lower mobility and the lower Seebeck coefficient reported in Ref. 1 . The effective mass, $m^{*}$ is calculated from the measured thermopower and carrier concentration, using the Fermi-Dirac integrals ${ }^{1,21,22}$

$$
\begin{gathered}
F_{r}\left(\eta_{F}\right)=\int_{0}^{\infty} \frac{u^{r} d u}{1+e^{\left(u-\eta_{F}\right)}}, \\
\alpha= \pm \frac{k_{B}}{e}\left[\frac{(2+r) F_{1+r}\left(\eta_{F}\right)}{F_{r}\left(\eta_{F}\right)}-\eta_{F}\right], \\
p=\frac{\left(2 m^{*} k_{B} T\right)^{3 / 2}}{2 \pi^{2} \hbar^{3}} F_{1 / 2}\left(\eta_{F}\right) .
\end{gathered}
$$

The reduced Fermi energy or $\eta_{F}\left(=\varepsilon_{F} / k_{B} T\right)$ is calculated from a known measured value of thermopower using Eq. (2), 


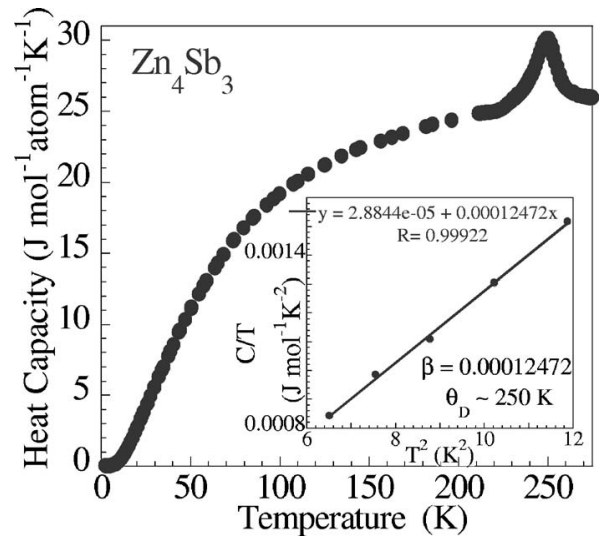

FIG. 2. Heat capacity of $\mathrm{Zn}_{4} \mathrm{Sb}_{3}$ approaches the Dulong Petit limit at high temperatures. The anomaly in the form of a peak at $250 \mathrm{~K}$ represents transformation from $\alpha$ to $\beta-\mathrm{Zn}_{4} \mathrm{Sb}_{3}$. Inset shows the low temperature fit for calculation of Debye temperature.

where we have used $r=0$. The effective mass, $m^{*}$, is then evaluated from Eq. (3) using the calculated $\eta_{F}$ and the measured carrier concentration, $p$. At $300 \mathrm{~K}$, the effective mass calculated from the thermopower $(\alpha \approx 160 \mu \mathrm{V} / \mathrm{K})$ and carrier concentration $\left(p \approx 3 \times 10^{19} \mathrm{~cm}^{-3}\right)$ yields $m^{*} \approx 0.9 m_{e}$. A hole drift mobility, $\mu_{H} \approx 70 \mathrm{~cm}^{2} \mathrm{~V}^{-1} \mathrm{~s}^{-1}$ is calculated using the relation $\sigma=p e \mu_{H}$, where $\sigma$ is the electrical conductivity, $p$ is the hole concentration, and $e$ is the electronic charge.

The heat capacity measurements, shown in Fig. 2, exhibit a well-defined peak at $250 \mathrm{~K}$ with no apparent thermal hysteresis, indicating a second order phase transition. The Debye temperature estimated from the low temperature region is $\Theta_{D} \approx 250 \mathrm{~K}$, which is in good agreement with the calculation of Caillat et al. ${ }^{1}$ The room temperature value of heat capacity is about $184 \mathrm{~J} / \mathrm{mol}-\mathrm{K}$ and approaches the Dulong-Petit limit of $3 R \mathrm{~J} / \mathrm{mol}$-atom- $\mathrm{K}$ at room temperature. The entropy of phase transition evaluated from integrating the $C p / T$ vs $T$ peak is $0.27 \mathrm{~J} / \mathrm{mol}$-atom- $\mathrm{K}$, which is attributed to the increased disorder of $\mathrm{Zn}$ atoms. Heat capacity measurements under an applied magnetic field of $5 \mathrm{~T}$ still reveal the peak in heat capacity at $250 \mathrm{~K}$, ruling out any magnetic transitions.

A distinct effect of the order-disorder phase transition is observed in the thermal conductivity at $250 \mathrm{~K}$ in Fig. 3. The $\alpha-\mathrm{Zn}_{4} \mathrm{Sb}_{3}$ exhibits a well-defined peak around $25 \mathrm{~K}$, after which the thermal conductivity decreases as $1 / T$, signifying an ordered crystal lattice with phonon scattering increasing with temperature. At $T \approx 250 \mathrm{~K}$, a kink is observed in the thermal conductivity of $\mathrm{Zn}_{4} \mathrm{Sb}_{3}$. The inset shows a change in slope with a decrease in thermal conductivity as the ordered $\alpha-\mathrm{Zn}_{4} \mathrm{Sb}_{3}$ changes to the disordered $\beta$ phase. The disorder in the crystal structure yields phonon scattering centers leading to a reduction in the lattice thermal conductivity of $\beta-\mathrm{Zn}_{4} \mathrm{Sb}_{3}$. The lattice contribution to thermal conductivity $\left(\kappa_{L}=\kappa_{T}-\kappa_{E}\right)$ is calculated using the Wiedemann-Franz relation $\left(\kappa_{E}=L_{O} \sigma T\right.$, where $L_{O}=2.45 \times 10^{-8} \mathrm{~V}^{2} / \mathrm{K}^{2}$, the Lorenz number). The total thermal conductivity $\left(\kappa_{T}\right)$ at $300 \mathrm{~K}$ is about $1.5 \mathrm{~W} \mathrm{~m}^{-1} \mathrm{~K}^{-1}$ (which is larger than that given in Ref. 1) and the calculated lattice contribution $\left(\kappa_{L}\right)$ is about $1.3 \mathrm{~W} \mathrm{~m}^{-1} \mathrm{~K}^{-1}$. Our value of $\kappa_{L}$ is in relatively good agreement with the theoretical predictions by Caillat et al. ${ }^{1}$ yield-
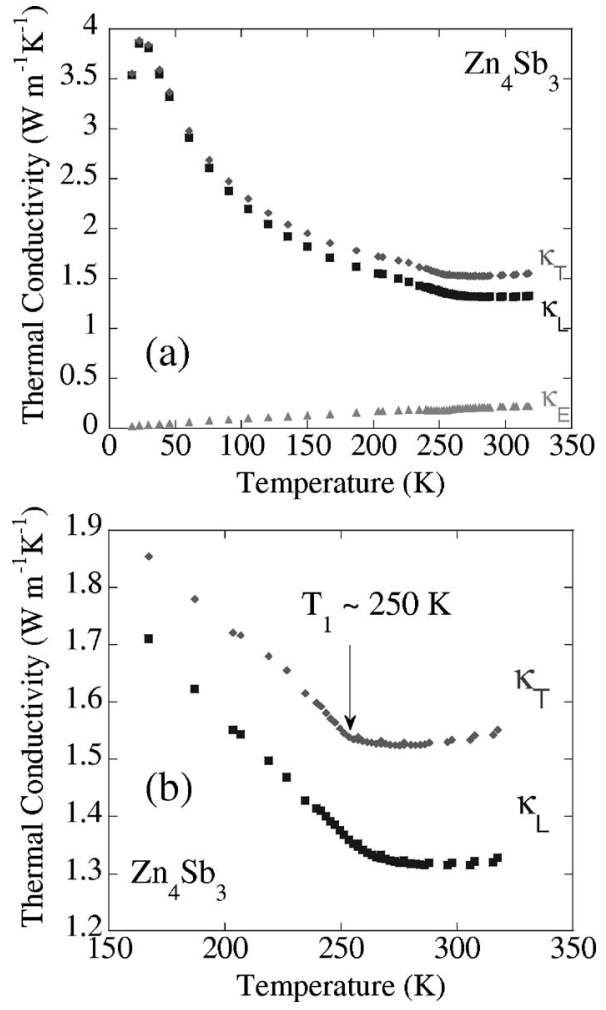

FIG. 3. (a) Thermal conductivity shows a change in slope at the phase transition $T_{1} \approx 250 \mathrm{~K}$, with a reduced thermal conductivity in the disordered $\beta$ phase. (b) The total $\left(\kappa_{T}\right)$ and lattice $\left(\kappa_{L}\right)$ contribution to thermal conductivity near the order-disorder phase transition.

ing a room temperature lattice thermal conductivity of about $1.14 \mathrm{~W} \mathrm{~m}^{-1} \mathrm{~K}^{-1}$. However, our lattice thermal conductivity is higher than the room temperature magnitude of $0.65 \mathrm{~W} \mathrm{~m}^{-1} \mathrm{~K}^{-1}$ reported by Caillat et al., ${ }^{1}$ possibly due to the fact that the samples reported here have less electronphonon scattering than those reported in Ref. 1, because of the lower carrier concentration. Our measurements have not been corrected for possible radiation losses.

It is interesting to note that the $\alpha-\mathrm{Zn}_{4} \mathrm{Sb}_{3}$ phase has a larger unit cell with a complex crystal structure and a larger number of symmetry nonequivalent atoms compared to the $\beta$ phase. It is expected that the larger unit cell with a complex structure would give the $\alpha$ phase a lower thermal conductivity compared to a simpler phase such as $\mathrm{ZnSb}$. Indeed, the thermal conductivity of $\mathrm{ZnSb}\left(2.7 \mathrm{~W} \mathrm{~m}^{-1} \mathrm{~K}^{-1}\right.$ at room temperature $)^{23}$ is nearly twice that of $\alpha-\mathrm{Zn}_{4} \mathrm{Sb}_{3}$. Yet, the disordered $\beta$ phase, with a small unit cell and fewer atoms has the lowest thermal conductivity compared to both $\alpha-\mathrm{Zn}_{4} \mathrm{Sb}_{3}$ and $\mathrm{ZnSb}$. This shows the relative importance of disorder in the reduction of thermal conductivity in a thermoelectric material in comparison to a material with a more complex crystal structure.

The elastic moduli of $\mathrm{Zn}_{4} \mathrm{Sb}_{3}$ are measured as a function of temperature using resonant ultrasound spectroscopy. Figure 4(a) shows the two elastic moduli as a function of temperature for polycrystalline $\mathrm{Zn}_{4} \mathrm{Sb}_{3}$, where $c_{11}$ is a compressional modulus and $c_{44}$ is the shear modulus. As the temperature increases from $4 \mathrm{~K}$ to about $150 \mathrm{~K}$, the observed temperature dependence of $c_{11}$ and $c_{44}$ can be fitted by the 

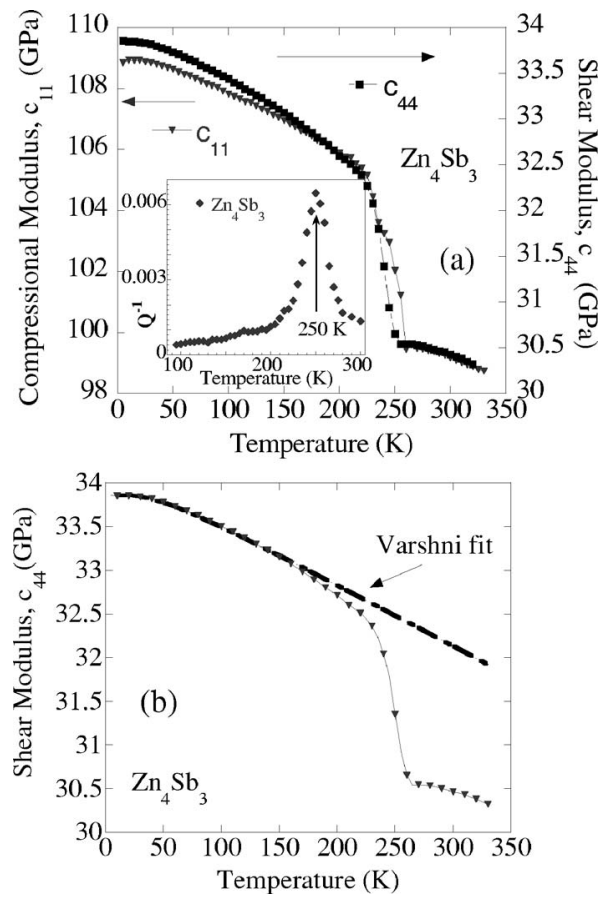

FIG. 4. (a) A dramatic effect of the structural phase transition at $T_{1} \approx 250 \mathrm{~K}$ on the elastic constants $c_{11}$ and $c_{44}$ of $\mathrm{Zn}_{4} \mathrm{Sb}_{3}$ measured using the RUS. Inset shows a peak in $Q^{-1}$ at the phase transition. (b) Varshni fit between 5 and $150 \mathrm{~K}$ indicating a "normal" temperature behavior of the elastic moduli before the dramatic lattice softening at the phase transition.

traditional Varshni function, ${ }^{24,25}$ an empirical function reported by Varshni to model the temperature dependence of elastic moduli for "ordinary" materials [Fig. 4(b)]. However, near the order-disorder phase transition at $250 \mathrm{~K}$, we observe a dramatic decrease in the elastic moduli of $\mathrm{Zn}_{4} \mathrm{Sb}_{3}$, with both $c_{11}$ and $c_{44}$ softening at the transition temperature. The $T$ dependence of the elastic moduli behavior around $250 \mathrm{~K}$ is typical for an order-disorder phase transition. ${ }^{26}$ In addition, the internal friction, $Q^{-1}=\Delta f / f$, has been determined from the full width at half maximum (FWHM) of the resonant frequencies, where $Q$ is the quality factor of the resonance. We observe a well-defined peak in $Q^{-1}$ at $T_{1}=250 \mathrm{~K}$ in the inset of Fig. 5(a), confirming the phase transition at this temperature. ${ }^{14}$ However, we do not see any evidence of a second phase transition at $234 \mathrm{~K}$ in our measurements.

The mean velocity of sound $\left(v_{m}\right)$ is calculated ${ }^{27}$ from 4 to $300 \mathrm{~K}$ with the measured values of $c_{11}$ and $c_{44}$ using $v_{L}=\sqrt{\frac{c_{11}}{\rho}}$ and $v_{T}=\sqrt{\frac{c_{44}}{\rho}}$. Our calculation of the mean sound velocity is in very good agreement with the velocity of sound at room temperature estimated by Caillat et al. ${ }^{1}$ The observed reduction in the sound velocity in Fig. 5(a), related to the softening of the lattice, may be attributed to soft acoustic phonon modes associated with an "instability" in the lattice at the phase transition. ${ }^{28,29}$

The typical expression for the phonon mean free path ${ }^{30}$ $\kappa_{L}=\frac{1}{3} c_{v} v_{m} \ell$ is derived from the kinetic theory of gases, where $\kappa_{L}$ is the lattice thermal conductivity, $c_{v}$ is the specific heat per unit volume of phonons, $\ell$ is the phonon mean free path, and $v_{m}$ is the phonon velocity (or the mean velocity of
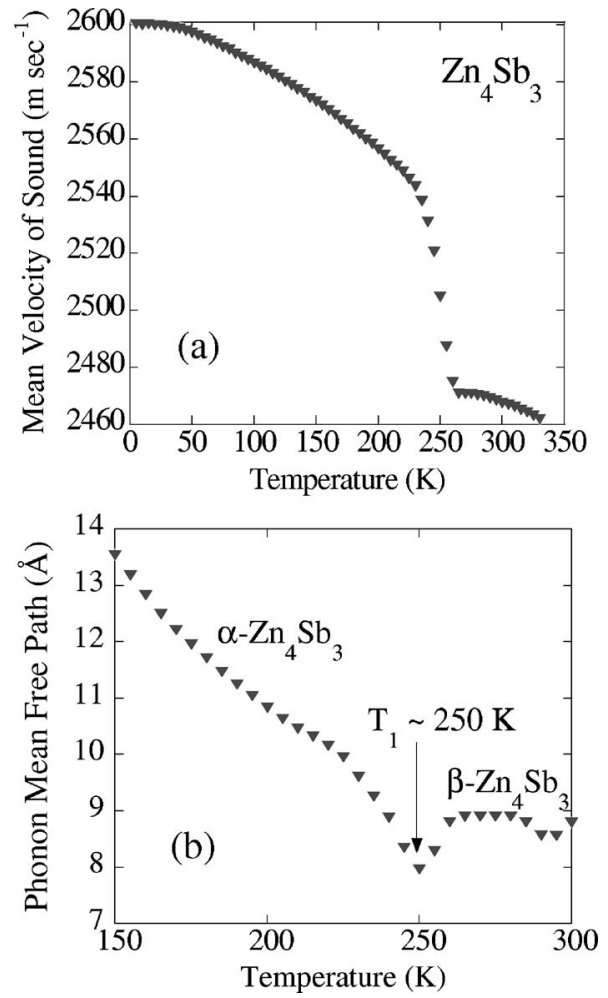

FIG. 5. (a) The mean velocity of sound calculated from the elastic moduli, $c_{11}$ and $c_{44}$. (b) Phonon mean free path shows a dip at the structural phase transition as $\mathrm{Zn}_{4} \mathrm{Sb}_{3}$ changes phase from $\alpha$ to the more disordered $\beta$ phase at $T_{1} \approx 250 \mathrm{~K}$.

sound through the material). Taking the frequency dependence of the sound velocity into account normally results in a much larger estimate of the average mean free path (50 to 100 times larger in Silicon). ${ }^{31}$ Nevertheless, Fig. 5(b) shows the phonon mean free path calculated using the typical method as a function of temperature. At about $150 \mathrm{~K}$, this phonon mean free path is about $14 \AA$ in $\alpha-\mathrm{Zn}_{4} \mathrm{Sb}_{3}$, which steadily decreases with increasing temperature, possibly due to umklapp scattering. Near $250 \mathrm{~K}$, there is a substantial dip in $\ell$ to about $8 \AA$ due to lattice softening at the structural phase transition. Above $250 \mathrm{~K}$, the phonon mean free path in $\beta-\mathrm{Zn}_{4} \mathrm{Sb}_{3}$ assumes a steady value of about $8.8 \AA$, the nearneighbor interatomic spacing on average being $2.7 \AA{ }^{9}$ The two different mechanisms of heat transfer in $\alpha-\mathrm{Zn}_{4} \mathrm{Sb}_{3}$ and $\beta-\mathrm{Zn}_{4} \mathrm{Sb}_{3}$ are apparent from the temperature dependence of the phonon mean free path, with a decrease in the lattice thermal conductivity in $\beta-\mathrm{Zn}_{4} \mathrm{Sb}_{3}$ pointing to an increased disorder in the $\beta$ phase.

\section{CONCLUSIONS}

We have presented a correlation between the thermal and elastic properties of $\mathrm{Zn}_{4} \mathrm{Sb}_{3}$ near the structural phase transition at $T_{1} \approx 250 \mathrm{~K}$. We observe dramatic effects of the orderdisorder phase transition of $\mathrm{Zn}_{4} \mathrm{Sb}_{3}$ on the elastic constants with a remarkable lattice softening at $250 \mathrm{~K}$. We also observe a change in slope and a reduction in the thermal conductivity in the disordered phase of $\beta-\mathrm{Zn}_{4} \mathrm{Sb}_{3}$. The dip in the phonon mean free path at the order-disorder phase transition 
followed by a reduction in $\ell$ in the $\beta$ phase indicates an increased phonon scattering leading to a decrease in the lattice thermal conductivity in the thermoelectric $\beta-\mathrm{Zn}_{4} \mathrm{Sb}_{3}$.

\section{ACKNOWLEDGMENTS}

We would like to acknowledge Franck Gascoin for his help in preparing the samples. We would also like to thank Brian Sales for valuable discussions. Work at The University of Tennessee is supported by the National Science Foundation under Grant No. DMR 0506292. The work at Clemson University was supported through DOE Grant No. DEFG02-04ER-46139 and SC EPSCoR.
*Contact author. Electronic address: sbhatta4@utk.edu

${ }^{\dagger}$ Present address: Institut für Festkörperforschung, Forschungszentrum Jülich GmbH, D-52425 Jülich, Germany.

${ }^{1}$ T. Caillat, J. P. Fleurial, and A. Borshchevsky, J. Phys. Chem. Solids 58, 7, 1119 (1997).

${ }^{2}$ V. Izard, M. C. Record, J. C. Tedenac, and S. G. Fries, CALPHAD: Comput. Coupling Phase Diagrams Thermochem. 25, 567 (2001).

${ }^{3}$ B. E. Curry, J. Phys. Chem. 13, 589-597 (1909).

${ }^{4}$ T. Takei, Sci. Rep. Res. Inst. Tohoku Univ. A 16, 1031 (1927).

${ }^{5}$ G. Vuillard and J. P. Piton, C. R. Acad. Sci., Ser. IIb: Mec., Phys., Chim., Astron. 263, 1018 (1966).

${ }^{6}$ Ya. A. Ugai, T. A. Marshakova, V. Ya. Shevchenko, and N. P. Demina, Inorg. Mater. 5, 1180 (1969).

${ }^{7}$ H. W. Mayer, I. Mikhail, and K. Schubert, J. Less-Common Met. 59, 43 (1978).

${ }^{8}$ J. Nylen, M. Andersson, S. Lidin, and U. Häussermann, J. Am. Ceram. Soc. 126, 16306 (2004).

${ }^{9}$ G. J. Snyder, M. Christensen, E. Nishibori, T. Caillat, and B. B. Iversen, Nat. Mater. 3, 458 (2004).

${ }^{10}$ F. Cargnoni, E. Nishibori, P. Rabiller, L. Bertini, G. J. Snyder, M. Christensen, C. Gatti, and B. B. Iversen, Chem.-Eur. J. 10, 3861 (2004).

${ }^{11}$ S. Kim, I. I. Mazin, and D. J. Singh, Phys. Rev. B 57, 11, 6199 (1988).

${ }^{12}$ A. L. Pope, R. T. Littleton IV, and T. M. Tritt, Rev. Sci. Instrum. 72, 1770 (2001).

${ }^{13}$ A. L. Pope, B. Zawilski, and T. M. Tritt, Cryogenics 41, 725 (2001).

${ }^{14}$ A. Migliori and John L. Sarrao, in Resonant Ultrasound Spectroscopy (John Wiley \& Sons, Inc., New York, 1997).

${ }^{15}$ A. Migliori, J. L. Sarrao, W. M. Visscher, T. M. Bell, M. Lei, Z.
Fisk, and R. G. Leisure, Physica B 183, 1 (1993).

${ }^{16}$ J. Maynard, Physics Today, p. 26, Jan. (1996).

${ }^{17}$ Y. Mozharivskyj, A. O. Pecharsky, S. Bud'ko, and G. J. Miller, Chem. Mater. 16, 1580 (2004).

${ }^{18}$ T. Souma, G. Nakamoto, and M. Kurisu, J. Alloys Compd. 340, 275 (2002).

${ }^{19}$ Y. Mozharivskyj, Y. Janssen, J. L. Harringa, A. Kracher, A. O. Tsokol, and G. J. Miller, Chem. Mater. 18, 822 (2006).

${ }^{20}$ R. D. Barnard, in Thermoelectricity in Metals and Alloys (Taylor and Francis LTD, London, 1972).

${ }^{21}$ G. A. Lamberton, Jr., S. Bhattacharya, R. T. Littleton, IV, M. A. Kaeser, R. H. Tedstrom, and T. M. Tritt, Appl. Phys. Lett. 80, 598 (2002).

${ }^{22}$ Neil Ashcroft and N. David Mermin, in Solid State Physics, Brooks Cole, 1st edition.

${ }^{23}$ R. C. Miller, Zinc Antimonide, in Thermoelectricity: Science and Engineering, edited by R. R. Heikes and R. W. Ure (Interscience, New York, 1961), pp. 405-407.

${ }^{24}$ Y. P. Varshni, Phys. Rev. B 2, 10, 3952 (1970).

${ }^{25}$ V. Keppens, D. Mandrus, B. C. Sales, B. C. Chakoumakos, P. Dai, R. Coldea, M. B. Maple, D. A. Gajewski, E. J. Freeman, and S. Bennington, Nature (London) 395, 876 (1998).

${ }^{26}$ W. Rehwald, Adv. Phys. 22(6), 721 (1973).

${ }^{27}$ O. L. Anderson, J. Phys. Chem. Solids 24, 909 (1963).

${ }^{28}$ Minoru Fujimoto, in The Physics of Structural Phase Transitions (Springer, New York, 2005), 2nd edition.

${ }^{29}$ P. J. Ford, W. A. Lambson, A. J. Miller, G. A. Saunders, H. Bach, and S. Methfessel, J. Phys. C 13, L697 (1980).

${ }^{30}$ H. M. Rosenberg, in The Solid State (Oxford Science Publications, New York, 1988), 3rd edition.

${ }^{31} \mathrm{C}$. Dames and G. Chen in CRC Handbook of Thermoelectrics (2005). 